\title{
Design of Two-Dimensional Recursive Filters Using Genetic Algorithms
}

\author{
Nikos E. Mastorakis, Senior Member, IEEE, Ioannis F. Gonos, Student Member, IEEE, and \\ M. N. S. Swamy, Life Fellow, IEEE
}

\begin{abstract}
In this paper, we examine a new design method for two-dimensional (2-D) recursive digital filters using genetic algorithms (GAs). The design of the 2-D filter is reduced to a constrained minimization problem the solution of which is achieved by the convergence of an appropriate GA. Theoretical results are illustrated by a numerical example. Also, comparison with the results of some previous design methods is attempted.
\end{abstract}

Index Terms-Constrained optimization, genetic algorithm (GA), multidimensional systems, two-dimensional (2-D) recursive filters, 2-D systems.

\section{INTRODUCTION}

D URING the last three decades, two-dimensional (2-D) filter design has received growing attention by researchers and practitioners working in image processing, biomedical imaging, seismic data processing, X-rays enhancement, satellite image processing, astronomy, etc. An overview is given in [1]-[3]. The design approaches for 2-D filters can be broadly classified into two categories:

1) those based on appropriate transformation of 1-D filters [2], [3];

2) those based on appropriate optimization techniques [3]-[10].

However, most of the existing algorithms [3]-[10] may result in an unstable filter. Various techniques have been proposed in order to overcome these instability problems, but the outcome is likely to be a system that has a very small stability margin and therefore may not be of practical importance [11].

In this paper, an optimization procedure is proposed by using the so-called evolutionary computation, or genetic algorithm (GA). The desired stability of 2-D filter yields appropriate constraints for the minimization problem. GAs are computational models inspired from biological evolution, which is based on natural selection. Thus, GAs are search algorithms based on the mechanics of natural genetics and natural selection. The backbone of every GA is the reproduction of an original population,

Manuscript received December 12, 2001; revised April 2, 2002. This paper was recommended by Associate Editor W.-P. Zhu.

N. Mastorakis is with the Hellenic Naval Academy, Department of Computer Science, Military Institutions of University Education, 18539 Piraeus, Greece (mastorakis@ieee.org).

I. F. Gonos was with the Hellenic Naval Academy, Department of Computer Science, Military Institutions of University Education, 18539 Piraeus, Greece. $\mathrm{He}$ is with the High Voltage Laboratory, National Technical University of Athens, 15780 Athens, Greece (igonos@ ieee.org).

M. N. S. Swamy is with the Center for Signal processing and Communications, Department of Electrical and Computer Engineering, Concordia University, Montreal, QC H3G IM8, Canada (e-mail: swamy@ece.concordia.ca).

Digital Object Identifier 10.1109/TCSI.2003.811019 the performance of crossover and mutation and the selection of the best. Artificial reproduction schemes were first developed in the seventies [12] and more were extended during the eighties [13], [14]. The search area for the GAs is very wide and it usually converges to a point near the global optimum. Fine tuning operations like hill climbing can be used to further refine the near-optimum solution. Simulating the survival of the most suitable among string structures, the optimal string (solution) is searched by randomized information exchange. Since the GA utilizes the coded discrete information of the artificial strings, it can be applied to ill-structured discrete optimization problems as well as to continuous optimization problems. They are often used in optimization problems, since they are capable of finding solutions to complex problems without carrying out an exhaustive search. Therefore, GAs are very often employed in nonlinear problems and multi-objective optimizations.

\section{Problem Formulation}

For design purposes, we consider the following 2-D transfer function for our filter:

$$
H\left(z_{1}, z_{2}\right)=H_{0} \frac{\sum_{i=0}^{K} \sum_{j=0}^{K} a_{i j} z_{1}^{i} z_{2}^{j}}{\prod_{k=1}^{K}\left(1+b_{k} z_{1}+c_{k} z_{2}+d_{k} z_{1} z_{2}\right)}, a_{00}=1 .
$$

Let us consider $M_{d}$, the desirable amplitude response of the 2-D filter as a function of the frequencies $\omega_{1}$ and $\omega_{2}$, $\left(\omega_{1}, \omega_{2} \in[0, \pi]\right)$. The design task at hand amounts to finding a transfer function $H\left(z_{1}, z_{2}\right)$ as in (1) such that the function $H\left(e^{j w_{1}}, e^{j w_{2}}\right)$ approximates the desired amplitude response $M_{d}\left(\omega_{1}, \omega_{2}\right)$. This approximation can be achieved by minimizing [11]

$$
\begin{aligned}
J & =J\left(a_{i j}, b_{k}, c_{k}, d_{k}, H_{0}\right) \\
& =\sum_{n_{1}=0}^{N_{1}} \sum_{n_{2}=0}^{N_{2}}\left[\left|M\left(\omega_{1}, \omega_{2}\right)\right|-M_{d}\left(\omega_{1}, \omega_{2}\right)\right]^{p}
\end{aligned}
$$

where

$$
M\left(\omega_{1}, \omega_{2}\right)=H\left(z_{1}, z_{2}\right) \mid \begin{aligned}
& z_{1}=e^{-j \omega_{1}} \\
& z_{2}=e^{-j \omega_{2}}
\end{aligned}
$$

and

$\omega_{1} \quad\left(\pi / N_{1}\right) n_{1}$

$\omega_{2} \quad\left(\pi / N_{2}\right) n_{2}$

$p \quad$ even positive integer (usually $p=2$ or $p=4$ ). 
Equation (2) is equivalent to

$$
J=\sum_{n_{1}=0}^{N_{1}} \sum_{n_{2}}^{N_{2}}\left[\left|M\left\{\frac{\pi n_{1}}{N_{1}}, \frac{\pi n_{2}}{N_{2}}\right\}\right|-M_{d}\left\{\frac{\pi n_{1}}{N_{1}}, \frac{\pi n_{2}}{N_{2}}\right\}\right]^{p} .
$$

Hence, the aim is to minimize the difference between the actual and desired amplitude response of the filter at $N_{1} N_{2}$ points. Since we are dealing with only first-degree factors in the denominator, it is known that the stability conditions are given by [1]-[3]

$$
\left|b_{k}+c_{k}\right|-1<d_{k}<1-\left|b_{k}-c_{k}\right|, \quad k=1,2, \ldots, K
$$

or

$$
\begin{array}{ll}
\left|b_{k}+c_{k}\right|-1<d_{k}, & k=1,2, \ldots, K \\
d_{k}<1-\left|b_{k}-c_{k}\right|, & k=1,2, \ldots, K .
\end{array}
$$

Thus, the design of 2-D recursive filters is equivalent to the following constrained minimization problem:

$$
\begin{aligned}
& \text { Minimize } J \\
& =\sum_{n_{1}=0}^{N_{1}} \sum_{n_{2}=0}^{N_{2}}\left[\left|M\left\{\frac{\pi n_{1}}{N_{1}}, \frac{\pi n_{2}}{N_{2}}\right\}\right|-M_{d}\left\{\frac{\pi n_{1}}{N_{1}}, \frac{\pi n_{2}}{N_{2}}\right\}\right]^{p}
\end{aligned}
$$

subject to the constraints

$$
\begin{array}{ll}
\left|b_{k}+c_{k}\right|-1<d_{k}, & k=1,2, \ldots, K \\
d_{k}<1-\left|b_{k}-c_{k}\right|, & k=1,2, \ldots, K .
\end{array}
$$

where $p$ is an even positive integer (usually $p=2$ or $p=4$ ), and $N_{1}, N_{2}$, and $K$ are given positive integers. In [11], this problem has been tackled using neural networks. In this paper, we give a solution using GAs. We will show the present method yields a better solution.

\section{PROBLEM SOLUTION}

For the purpose of illustration, without loss of generality, we consider the case of $K=2$. Then, $H\left(z_{1}, z_{2}\right)$ from (1) is as shown in (7) at the bottom of the page. For a successful implementation of the GA, we need to express $\left|M\left(\omega_{1}, \omega_{2}\right)\right|$ and the constraints given by (5) in appropriate forms. From (7) and (3), we obtain (8a) shown at the bottom of the page, where

$$
\begin{gathered}
c_{p q}=c_{p q}\left(\omega_{1}, \omega_{2}\right)=\cos \left(p \omega_{1}+q \omega_{2}\right) \\
s_{p q}=s_{p q}\left(\omega_{1}, \omega_{2}\right)=\sin \left(p \omega_{1}+q \omega_{2}\right), \\
p, q=0,1,2
\end{gathered}
$$

and

$$
\begin{aligned}
D= & {\left[\left(1+b_{1} c_{10}+c_{1} c_{01}+d_{1} c_{11}\right)\right.} \\
& \left.-j\left(b_{1} s_{10}+c_{1} s_{01}+d_{1} s_{11}\right)\right] * \\
& *\left[\left(1+b_{2} c_{10}+c_{2} c_{01}+d_{2} c_{11}\right)\right. \\
& \left.-j\left(b_{2} s_{10}+c_{2} s_{01}+d_{2} s_{11}\right)\right] .
\end{aligned}
$$

In a compact form, $M\left(\omega_{1}, \omega_{2}\right)$ can be written as

$$
M\left(\omega_{1}, \omega_{2}\right)=H_{0} \frac{A_{R}-j A_{I}}{\left(B_{1 R}-j B_{1 I}\right)\left(B_{2 R}-j B_{2 I}\right)}
$$

where

$$
\begin{aligned}
A_{R}= & a_{00}+a_{01} c_{01}+a_{02} c_{02}+a_{10} c_{10}+a_{20} c_{20} \\
& +a_{11} c_{11}+a_{12} c_{12}+a_{21} c_{21}+a_{22} c_{22} \\
A_{I}= & a_{01} s_{01}+a_{02} s_{02}+a_{10} s_{10}+a_{20} s_{20}+a_{11} s_{11} \\
& +a_{12} s_{12}+a_{21} s_{21}+a_{22} s_{22} \\
B_{1 R}= & \left(1+b_{1} c_{10}+c_{1} c_{01}+d_{1} c_{11}\right) \\
B_{1 I}= & \left(b_{1} s_{10}+c_{1} s_{01}+d_{1} s_{11}\right) \\
B_{2 R}= & \left(1+b_{2} c_{10}+c_{2} c_{01}+d_{2} c_{11}\right) \\
B_{2 I}= & \left(b_{2} s_{10}+c_{2} s_{01}+d_{2} s_{11}\right) .
\end{aligned}
$$

Therefore, we have

$$
\left|M\left(\omega_{1}, \omega_{2}\right)\right|=H_{0} \frac{\sqrt{A_{R}^{2}+A_{I}^{2}}}{\left(B_{1 R}^{2}+B_{1 I}^{2}\right)\left(B_{2 R}^{2}+B_{2 I}^{2}\right)}
$$

A continuous differentiable form of the constraints can be obtained from (5) in the form

$$
\begin{aligned}
-\left(1+d_{k}\right) & <\left(b_{k}+c_{k}\right)<\left(1+d_{k}\right) \\
-\left(1-d_{k}\right) & <\left(b_{k}-c_{k}\right)<\left(1-d_{k}\right) \\
\left(1+d_{k}\right) & >0 \\
\left(1-d_{k}\right) & <0 .
\end{aligned}
$$

Let us suppose that the desired amplitude response is given by

$$
M_{d}\left(\omega_{1}, \omega_{2}\right)= \begin{cases}1, & \text { if } \sqrt{\omega_{1}^{2}+\omega_{2}^{2}} \leq 0.12 \\ 0.5, & \text { if } 0.08 \pi \leq \sqrt{\omega_{1}^{2}+\omega_{2}^{2}} \leq 0.12 \\ 0, & \text { otherwise. }\end{cases}
$$

$$
H\left(z_{1}, z_{2}\right)=H_{0} \frac{a_{00}+a_{01} z_{2}+a_{02} z_{2}^{2}+a_{10} z_{1}+a_{20} z_{1}^{2}+a_{11} z_{1} z_{2}+a_{12} z_{1} z_{2}^{2}+a_{21} z_{1}^{2} z_{2}+a_{22} z_{1}^{2} z_{2}^{2}}{\left(1+b_{1} z_{1}+c_{1} z_{2}+d_{1} z_{1} z_{2}\right)\left(1+b_{2} z_{1}+c_{2} z_{2}+d_{2} z_{1} z_{2}\right)}
$$

$$
M\left(\omega_{1}, \omega_{2}\right)=H_{0}\left[\begin{array}{c}
\frac{\left[a_{00}+a_{01} c_{01}+a_{02} c_{02}+a_{10} c_{10}+a_{20} c_{20}+a_{11} c_{11}+a_{12} c_{12}+a_{21} c_{21}+a_{22} c_{22}\right]}{D} \\
-\frac{j\left[a_{01} s_{01}+a_{02} s_{02}+a_{10} s_{10}+a_{20} s_{20}+a_{11} s_{11}+a_{12} s_{12}+a_{21} s_{21}+a_{22} s_{22}\right]}{D}
\end{array}\right]
$$


Choosing the values $p=2, N_{1}=50$, and $N_{2}=50$, the corresponding constrained optimization problem (6) becomes

$$
\begin{aligned}
& \text { Minimize } J \\
& =\sum_{n_{1}=0}^{50} \sum_{n_{2}=0}^{50}\left[\left|M\left(\frac{\pi}{50} n_{1}, \frac{\pi}{50} n_{2}\right)\right|-M_{d}\left(\frac{\pi}{50} n_{1}, \frac{\pi}{50} n_{2}\right)\right]^{2}
\end{aligned}
$$

subject to the constraints given by (12) with $k=1,2$. The same problem has been tackled in [11] using neural networks. In this paper, we give a solution using GAs, which has the advantage of producing a smaller error. To start implementing a GA, an initial population is considered. This is represented by "bit" strings that evolve randomly through successive generations in order to obtain an optimum value for a particular fitness function [13]. In each generation, a new set of artificial strings is created using bits and pieces of the most suitable of the old ones. Solutions with high suitability are mated with other solutions by crossing over parts of solution strings. Strings may also mutate. Solutions with poor fitness are improved by crossover using highly fit solutions.

A simple GA relies on the processes of reproduction, crossover and mutation to reach the global or "near-global" optimum. To start the search, GAs require an initial set of points. This set is called population, analogous to the biological system. It has a population size $P_{s}$. A random number generator creates the initial population. This initial set is converted to a binary system and is considered as chromosomes, actually sequences of " 0 " and " 1 ". The next step is to form pairs of these points that will be considered as parents for a reproduction. Parents come to reproduction and interchange $N_{p}$ parts of their genetic material. This is achieved by crossover. After the crossover, there is a very small probability $P_{m}$ for mutation. Mutation is the phenomenon where a random " 0 " becomes " 1 " or a " 1 " becomes " 0 ". Mutation is necessary because although reproduction and crossover efficiently search and mix existing strings, occasionally they may result in loss of some potentially useful "genetic" material. Assume that each pair of "parents" gives rise to $N_{c}$ children. Thus, the GA generates the initial layouts and obtains the objective function values. The above operations are carried out and the next generation with a new population of strings is formed. By the reproduction, the population of the "parents" is enhanced by the "children", increasing the original population since new members are added. The parents always belong to the population under consideration. The new population has now $P_{s}+N_{c} \cdot P_{s} / 2$ members. Then the process of natural selection is applied. According to this process only $P_{s}$ members survive out of the $P_{s}+N_{c} \cdot P_{s} / 2$ members. These $P_{s}$ members are selected as those members with the highest values of $J$, if we are attempting to maximize $J$, (or with the lowest values of $J$, if we are attempting to minimize $J)$. By repeated iterations of reproduction under crossover and mutation, and natural selection, we can find the maximum (or minimum) of $J$ as the point to which the best values of our population converge. The termination criterion is fulfilled if either the mean value of $J$ in the $P_{s}$-members population is no longer improved (maximized or minimized), or the number of iterations is greater than the maximum number of iterations $N_{\max }$, which is defined by us.

In the present problem under consideration, our aim is to minimize the vector $x$, where

$x=$

$\left(a_{00}, a_{02}, a_{01}, a_{11}, a_{12}, a_{20}, a_{21}, a_{22}, b_{1}, b_{2}, c_{1}, c_{2}, d_{1}, d_{2}, H_{0}\right)^{T}$.

Each coordinate of the vector is converted to the binary system and is considered as a part of a big chromosome. The search starts with a randomly generated population of such $P_{s}$ chromosomes. Each coefficient is converted to a $t$-bits binary number. Then, $2 t$ bits are required for the "chromosome" of each element with an absolute value less than 3. Our GA is summarized as follows.

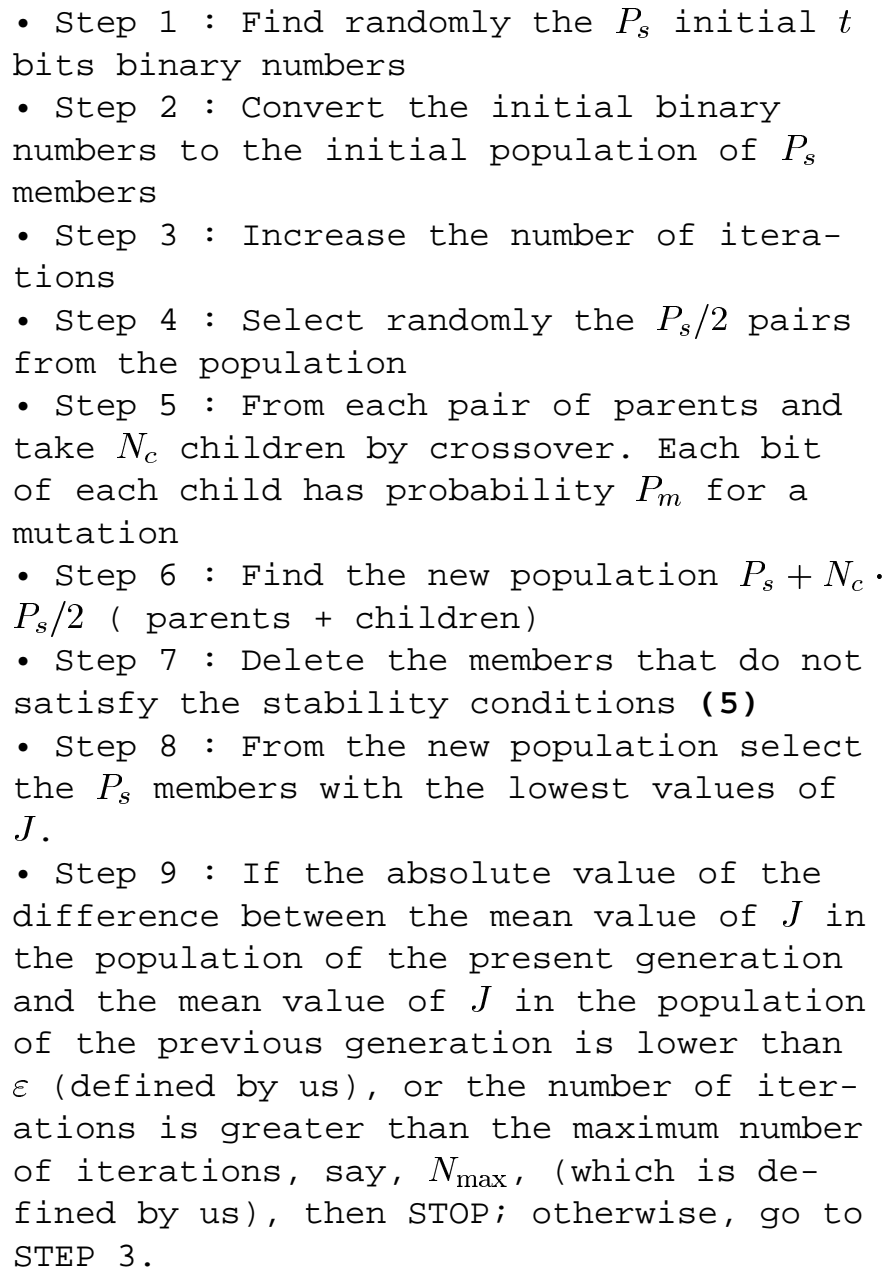

It is observed that the designed filter is stable because in Step 7 of the above GA the Stability conditions (12) are checked.

For better results, the above GA was run $P_{s}$ times. The $P_{s}$ best result of each time was kept and was the initial population for a new GA. The parameters of the GA are presented in Table I. 
TABLE I

PARAMETERS OF THE GA

\begin{tabular}{c|c|c|c|c|c}
\hline$P_{s}$ & $t$ & $N_{c}$ & $P_{m}$ & $N_{p}$ & $N_{\max }$ \\
\hline 500 & 32 & 10 & $5 \%$ & 12 & 200 \\
\hline
\end{tabular}

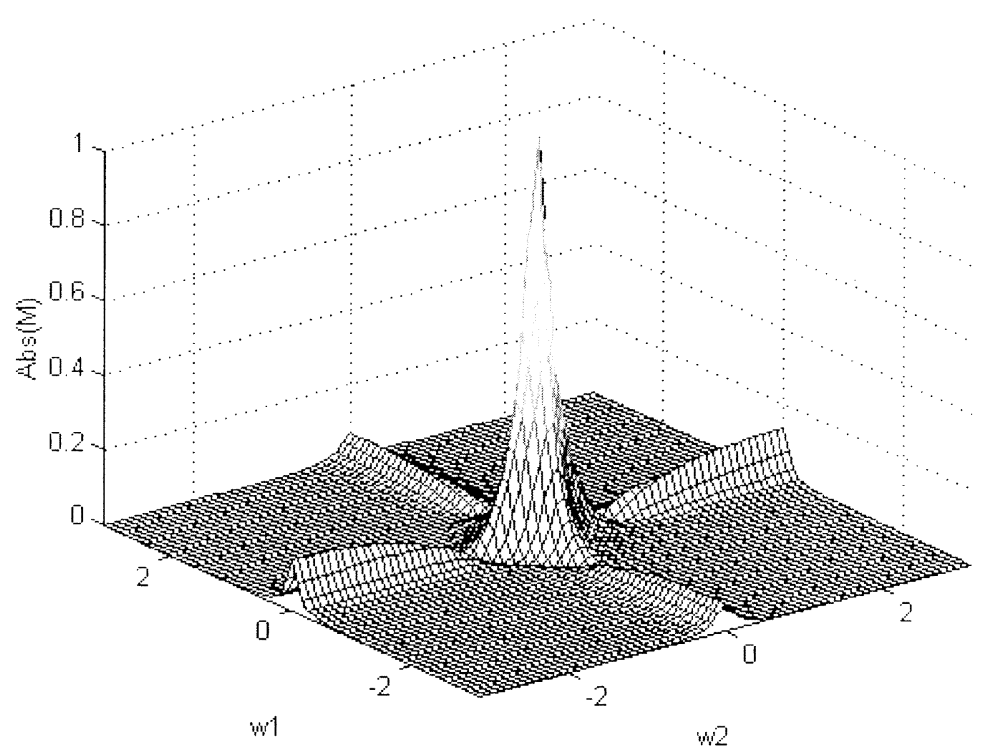

Fig. 1. Amplitude response $\left|M\left(\omega_{1}, \omega_{2}\right)\right|$ of the considered 2-D filter using GAs.

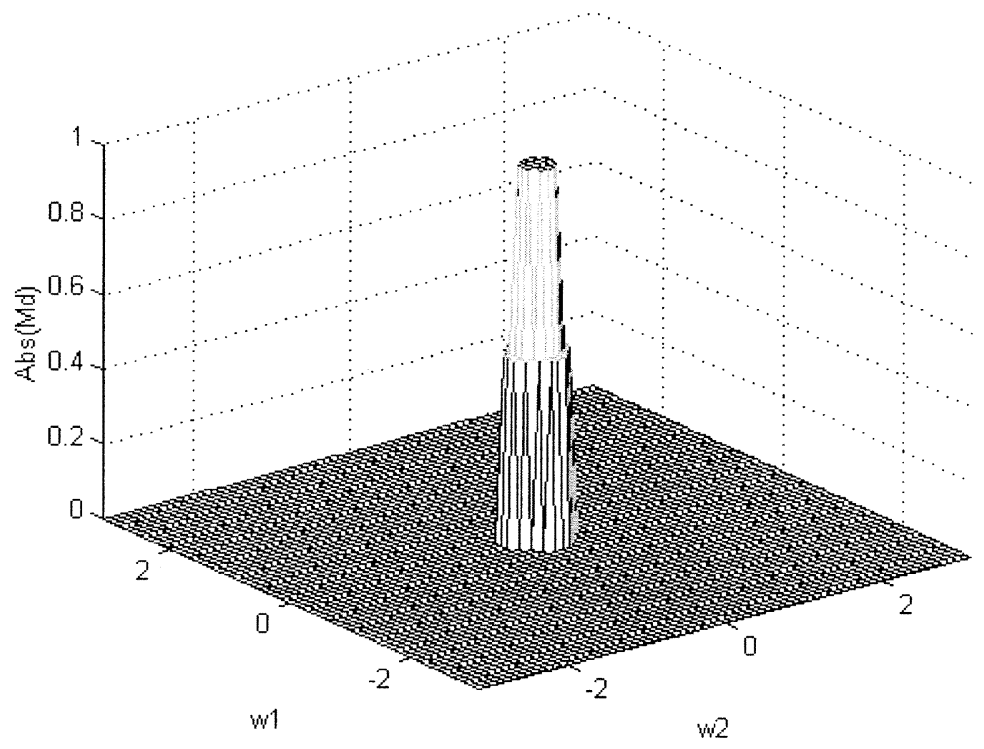

Fig. 2. Desired amplitude response $\left|M_{d}\left(\omega_{1}, \omega_{2}\right)\right|$ of the 2-D filter.

The vector $x$ obtained through the use of the GA described above is

$$
\begin{aligned}
x= & {[1.8162,-1.1060,0.0712,-2.5132,0.4279,} \\
& 0.5926,-1.3690,2.4326,-0.8662,-0.8907, \\
& -0.8531,-0.8388,0.7346,0.8025,0.0009]^{T} .
\end{aligned}
$$

Therefore, we get the equation shown at the bottom of the page. The corresponding amplitude response $\left|M\left(\omega_{1}, \omega_{2}\right)\right|$ is shown in Fig. 1, while Fig. 2 shows the desired amplitude response $\left|M_{d}\left(\omega_{1}, \omega_{2}\right)\right|$. Furthermore, for comparison, the result of the method given in [3] and [4] is presented in Fig. 3 and that of the method given in [11] is presented in Fig. 4.

$H\left(z_{1}, z_{2}\right)=0.009 \frac{1+0.0712 z_{1}+0.5926 z_{1}^{2}-2.5132 z_{1} z_{2}+0.4279 z_{1} z_{2}^{2}+1.8162 z_{2}+0.4279 z_{1}^{2} z_{2}+2.4326 z_{1}^{2} z_{2}^{2}-1.1060 z_{2}^{2}}{\left(1-0.8662 z_{1}-0.8531 z_{2}+0.7346 z_{1} z_{2}\right)\left(1-0.8907 z_{1}-0.8388 z_{2}+0.8025 z_{1} z_{2}\right)}$ 


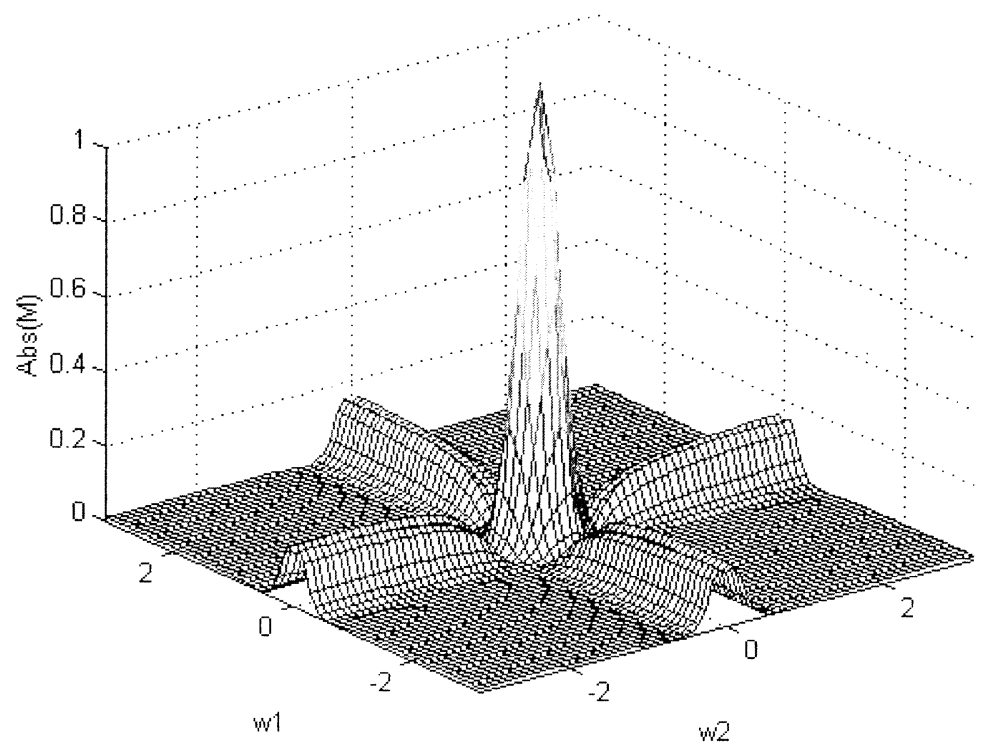

Fig. 3. Amplitude response $\left|M\left(\omega_{1}, \omega_{2}\right)\right|$ of the considered 2-D filter using the method in [3], [4].

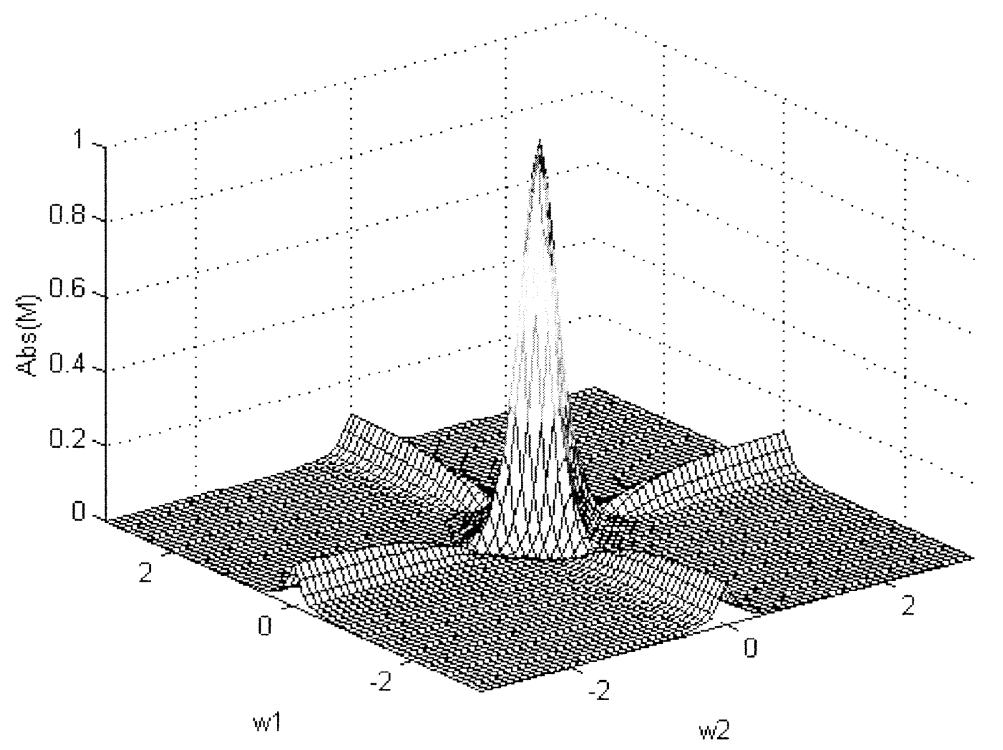

Fig. 4. Amplitude response $\left|M\left(\omega_{1}, \omega_{2}\right)\right|$ of the considered 2-D filter using the method in [11].

\section{CONCLUSION}

In this study, the design of 2-D recursive filters is attempted by using an appropriate evolutionary computational scheme. One of the main advantages of the present method over the method of [3] and [4] is that we can check the stability of the designed filter from the beginning of the procedure, since we introduce the desired stability as appropriate constraints. On the contrary, the previous methods, which are based more or less on a trial-and-error approach, cannot always guarantee the stability of the filter. Further, our implementation leads to a simpler filter since, in practice, we have to realize a factorable denominator and in particular of first-order filters, which obviously are simpler than those of [3], [4]. For the same numerical example, the present method finds a better approximation of the transfer function than the method of [11].

\section{REFERENCES}

[1] T. Kaczorek, Two-Dimensional Linear Systems. Berlin, Germany: Springer-Verlag, 1985.

[2] S. G. Tzafestas, Ed., Multidimensional Systems, Techniques and Applications. New York: Marcel Dekker, 1986.

[3] W.-S Lu and A. Antoniou, Two-Dimensional Digital Filters. New York: Marcel Dekker, 1992.

[4] G. A. Maria and M. M. Fahmy, "An lp design technique for two-dimensional digital recursive filters," IEEE Trans. Acoust., Speech, Signal Processing, vol. 22, pp. 15-21, Feb. 1974.

[5] C. Charalambous, "Design of 2-Dimensional circularly-symmetric digital filters," Proc. Inst. Elect. Eng., pt. G, vol. 129, pp. 47-54, Apr. 1982.

[6] P. K. Rajan and M. N. S. Swamy, "Quadrantal symmetry associated with two-dimensional digital transfer functions," IEEE Trans. Circuits Syst., vol. 29, pp. 340-343, June 1983.

[7] T. Laasko and S. Ovaska, "Design and implementation of efficient IIR notch filters with quantization error feedback," IEEE Trans. Instrum. Meas., vol. 43, pp. 449-456, June 1994. 
[8] C.-H. Hsieh, C.-M. Kuo, Y.-D. Jou, and Y.-L. Han, "Design of two-dimensional FIR digital filters by a two-dimensional WLS technique," IEEE Trans. Circuits Syst. II, vol. 44, pp. 348-412, May 1997.

[9] M. Daniel and A. Willsky, "Efficient implementations of 2-D noncausal IIR filters," IEEE Trans. Circuits Syst. II, vol. 44, pp. 549-563, July 1997.

[10] W.-P. Zhu, M. O. Ahmad, and M. N. S. Swamy, "A closed-form solution to the least-square design problem of 2-D linear-phase FIR filters," IEEE Trans. Circuits Syst. II, vol. 44, pp. 1032-1039, Dec. 1997.

[11] V. Mladenov and N. Mastorakis, "Design of two-dimensional recursive filters by using neural networks," IEEE Trans. Neural Networks, vol. 12, pp. 585-590, May 2001.

[12] H. Holland, Adaptation in Natural and Artificial Systems. Ann Arbor, MI: Univ. of Michigan Press, 1992.

[13] D. E. Goldberg, Genetic Algorithms in Search, Optimization, and Machine Learning. Reading, MA: Addison-Wesley, 1989.

[14] K. Krishnakumar and S. N. Melkote, "Machining fixture layout optimization using the genetic algorithm," Int. J. Machine Tools Manuf., vol. 40, pp. 579-598, 2000.

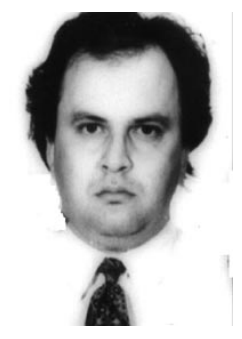

Nikos E. Mastorakis (M'97-SM'99) received the B.Sc. and M.Sc. degrees in electrical engineering, the $\mathrm{Ph} . \mathrm{D}$. degree in electrical engineering and computer science, and the B.Sc. degree (Ptychion) in pure mathematics from the National University of Athens, Athens, Greece.

He have served as a Special Scientist on Computers and Electronics in the Hellenic (Greek) Army General Staff (1993-1994) and taught courses in the Electrical Engineering Department of the National Technical University of Athens (1994-1998). He has also served as Visiting Professor at the School of Engineering, University of Exeter, Exeter, U.K. in 1998. He is currently a Professor and the Head of the Department of Computer Science at the Military Institutions of University Education (MIUE), Hellenic Naval Academy, Piraeus, Greece. He is an active researcher in applied mathematics and computer science (systems theory, control, optimization theory, algorithms theory, signal processing, robotics, computational intelligence). He has edited 33 books and authored five. He has over 200 published papers in international books, journals, and conferences. He has been a reviewer for 18 international journals and a Member of the Editorial Board of eight international journals including Advances in Computation: Theory and Practice, Editor of the International Book Series Electrical and Computer Engineering, Mathematics and Computers in Science and Engineering (World Scientific: Athens, Greece), the Editor-in-Chief of the International Journal of Computer Research, and International Journal of Communications and Networking. He has organized many international conferences, invited sessions and lectures.

Dr. Mastorakis is a member of the World Scientific and Engineering Academy and Society, the New York Academy of Sciences, the Armed Forces Communications and Electronics Association, and the American Association for the Advancement of Science. He is a Registered Professional Electrical and Mechanical Engineer. He has received several awards (Royal Society of England, Hellenic National Research Foundation, etc.) for his academic studies and his scientific research.

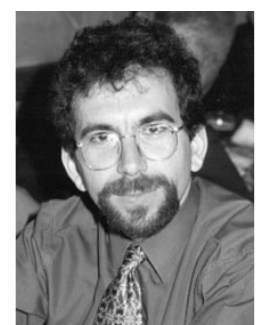

Ioannis F. Gonos (S'02) was born on May 8, 1970, in Artemisio, Arcadia, Greece. He received the diploma in electrical engineering and the Ph.D. degree from the National Technical University of Athens, Athens, Greece, in 1993 and 2002, respectively.

He was a Teaching Assistant at the Greek Naval Academy and the Technological Education Institute of Athens (1996-2001). He is currently a Researcher for the High Voltage Laboratory of the National Technical University of Athens. His research interests concern grounding system, insulators, high voltages, modeling, and genetics algorithms.

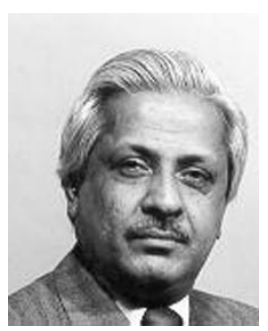

M. N. S. Swamy (S'59-M'62-SM'74-F'80-LF'01) received the B.Sc. (Hons.) degree in mathematics from Mysore University, Mysore, India, in 1954, the diploma in electrical communication engineering from the Indian Institute of Science, Bangalore, India, in 1957, and the M.Sc. and Ph. D. degrees in electrical engineering from the University of Saskatchewan, Saskatoon, SK, Canada, in 1960 and 1963, respectively

$\mathrm{He}$ is presently a Research Professor and the Director of the Center for Signal Processing and Communications in the Department of Electrical and Computer Engineering at Concordia University, Montreal, QC, Canada, where he served as the Chair of the Department of Electrical Engineering from 1970 to 1977, and Dean of Engineering and Computer Science from 1977 to 1993 . Since July 2001, he holds the Concordia Chair (Tier I) in Signal Processing. He has also taught in the Electrical Engineering Department of the Technical University of Nova Scotia, Halifax, NS, Canada, and the University of Calgary, Calgary, AB, Canada, as well as in the Department of Mathematics at the University of Saskatchewan. He has published extensively in the areas of number theory, circuits, systems and signal processing, and holds four patents. He is the coauthor of two book chapters and three books: Graphs, Networks and Algorithms (New York: Wiley, 1981), Graphs: Theory and Algorithms (New York: Wiley, 1992), and Switched Capacitor Filters: Theory, Analysis and Design (London, U.K. : Prentice Hall International Ltd., 1995). A Russian Translation of the first book was published by Mir Publishers, Moscow, U.S.S.R., in 1984, while a Chinese version was published by the Education Press, Beijing,China, in 1987.

Dr. Swamy is a Fellow of the Institute of Electrical Engineers (U.K.), the Engineering Institute of Canada, the Institution of Engineers (India), and the Institution of Electronic and Telecommunication Engineers (India). Presently, he is President-Elect of the Circuits and Systems (CAS) Society. He has served the IEEE in various capacities such as the Vice-President of the CAS society in 1976, Vice-President (Publications) of the CAS Society during 2001-2002, Editor-in-Chief of the IEEE TRANSACTIONS ON CIRCUITS AND SySTEMS-I: FundAMENTAL THEORY AND APPLICATIONS during 1999-2001, Associate Editor of the IEEE TRANSACTIONS ON CIRCUITS AND SySTEMS during 1985-1987, Program Chair for the 1973 IEEE CAS Symposium, General Chair for the 1984 IEEE CAS Symposium, Vice-Chair for the 1999 IEEE CAS Symposium, and a member of the Board of Governors of the CAS Society. He is the recipient of many IEEE-CAS Society awards including the Education Award in 2000, Golden Jubilee Medal in 2000, and the 1986 Guillemin-Cauer Best Paper Award. In August 2001, he was awarded the Doctor of Science in Engineering (Honoris Causa) by Ansted University "In recognition of his exemplary contributions to the research in Electrical and Computer Engineering and to Engineering Education, as well as his dedication to the promotion of Signal Processing and Communications Applications". $\mathrm{He}$ is a member of Micronet, a National Network of Centers of Excellence in Canada, and also its coordinator for Concordia University. 